\title{
Threshold resummation of the rapidity distribution for Drell-Yan production at $\mathrm{NNLO}+\mathrm{NNLL}$
}

\author{
Pulak Banerjee, ${ }^{1,2, *}$ Goutam Das, ${ }^{3, \dagger}$ Prasanna K. Dhani, ${ }^{1,2, \$}$ and V. Ravindran ${ }^{1,2, \S}$ \\ ${ }^{1}$ The Institute of Mathematical Sciences, Taramani, Chennai 600113, India \\ ${ }^{2}$ Homi Bhabha National Institute, Training School Complex, Anushakti Nagar, Mumbai 400085, India \\ ${ }^{3}$ Theory Group, Deutsches Elektronen-Synchrotron (DESY), \\ Notkestrasse 85, D-22607 Hamburg, Germany
}

(Received 20 May 2018; published 19 September 2018)

\begin{abstract}
We consider the production of pairs of lepton through the Drell-Yan process at the LHC and present the most accurate prediction on their rapidity distribution. While the fixed order prediction is already known to next-to-next-to-leading order in perturbative QCD, the resummed contribution coming from threshold region of phase space up to next-to-next-to-leading logarithmic (NNLL) accuracy has been computed in this article. The formalism developed in [1-3] has been used to resum large threshold logarithms in the two dimensional Mellin space to all orders in perturbation theory. We have done a detailed numerical comparison against other approaches that resum certain threshold logarithms in Mellin-Fourier space. Our predictions at NNLL level are close to theirs even though at leading logarithmic and next-to-leading logarithmic level we differ. We have also investigated the impact of these threshold logarithms on the stability of perturbation theory against factorization and renormalization scales. While the dependence on these scales does not get better with resummed results, the convergence of the perturbative series shows a better trend compared to the fixed order predictions. This is evident from the reduction in the K-factor for the resummed case compared to fixed order. We also present the uncertainties on the predictions resulting from parton distribution functions.
\end{abstract}

DOI: 10.1103/PhysRevD.98.054018

\section{INTRODUCTION}

The standard model (SM) has been extremely successful in describing the physics of elementary particles. The production of oppositely charged lepton-pairs, known as the Drell-Yan production [4], is one of the benchmark processes to probe physics at $\mathrm{TeV}$ energies at the colliders, namely earlier at Tevatron and now at the Large Hadron Collider (LHC). Because of its large cross section and small systematic uncertainties, Drell-Yan production also serves as luminosity monitor [5] at the LHC. Most importantly, at hadron colliders, Drell-Yan production provides valuable information about the partonic structure of hadrons, its clean electromagnetic probe is best suited for the search of any new physics beyond the SM (BSM). An excess rate over the SM in this channel will potentially indicate the

\footnotetext{
*bpulak@imsc.res.in

†outam.das@desy.de

prasannakd@imsc.res.in

\$ravindra@imsc.res.in
}

Published by the American Physical Society under the terms of the Creative Commons Attribution 4.0 International license. Further distribution of this work must maintain attribution to the author(s) and the published article's title, journal citation, and DOI. Funded by SCOAP. signature of BSM physics. Drell-Yan is the potential background for processes involving $Z^{\prime}$ or $W^{\prime}$ and also for spin-2/graviton searches. With the current LHC-13 and upcoming LHC-14 runs, more events will be available to precisely study the Drell-Yan distributions over a wide kinematic region.

Due to its undeniable importance, Drell-Yan has been studied theoretically to a great extent over many decades [6,7]. There has been continuous efforts towards the computation of higher order QCD and electroweak (EW) corrections to unprecedented accuracy. The full inclusive production cross section is known up to next-to-next-toleading order (NNLO) $[8,9]$ for a very long time. Very recently, predictions at next-to-next-to-next-to-leadingorder $\left(\mathrm{N}^{3} \mathrm{LO}\right)$ level have become available considering only the dominant soft-virtual (SV) contributions [10,11]. Electroweak corrections beyond leading-order (LO) are also known and at next-to-leading-order (NLO) level, they were computed in $[12,13]$. While inclusive production is important for precise prediction of cross section, differential distributions allow a wider comparison with experiments. Fully differential distributions such as rapidity, transverse momentum for the Drell-Yan are known to up to NNLO level in QCD [14-18]. SV contributions for the rapidity distribution are also known at $\mathrm{N}^{3} \mathrm{LO}$ level $[2,19,20]$. 
Studies where both QCD and EW corrections are combined can be found in [21]. Parton showers matched with NLO QCD results for the Drell-Yan are also available in MC@NLO [22], POWHEG [22,23] and aMC@NLO [24] frameworks.

One of the differential distributions that has been studied extensively is the transverse momentum $\left(p_{T}\right)$ distribution of pair of leptons or the vector bosons such as $Z / W^{ \pm}$, see [25-29], often in their large $p_{T}$ region. The rapidity distribution in Drell-Yan was computed in [25] at NLO level in QCD and it was then extended to NNLO level in [14,17] which stabilize the predictions [15] giving only a few percentage sensitivity to renormalization and factorization scales, say for example at the $Z$ mass region. However it has to be noted that the result does vary significantly with respect to the choices of different parton distribution functions (PDFs). In particular, at large invariant mass or at large rapidity of the final state, the cross sections are sensitive to large Björken $x$ regions of PDFs, where different PDFs show not only differences between them but also exhibit large uncertainties. For a recent review see [30]. This sensitivity of PDFs will in turn help to constraint the PDF sets much better. Hence, it is important to study these distributions. Certain distributions in Drell-Yan production also helps to study unpolarized transverse momentum dependent PDFs. For the recent developments, see [31,32].

The fixed order predictions are often not reliable in certain regions of phase space where large logarithms of some kinematic variables can appear. For example, at the partonic threshold, i.e., where the initial partons have just enough energy to produce the final state such as a pair of leptons or $Z / W^{ \pm}$and soft gluons, the phase space available for the gluons become severely constrained which results in large logarithms. These large logarithms however can be systematically resummed to all orders in perturbation theory for reliable predictions. This has made the resummation program an important topic of investigation over many years. For the inclusive production, the resummation of soft gluons in the threshold region was established [1,33-38] in the Mellin space and for the transverse momentum distribution, at small $p_{T}$, the resulting large logarithms were shown to exponentiate in the impact parameter space $[39,40]$. A modern approach based on soft-collinear effective theory (SCET) demonstrates similar resummation in momentum space, see [41] for inclusive production and [42] for transverse momentum distribution. Resummation for the differential distribution with respect to the Feynman variable $x_{F}$ which describes the longitudinal momentum of the final state was studied in [1] and it was found that there were two thresholds and both could be resummed to all orders. For the resummation with respect to $x_{F}$ using a different scheme see [43]. For the rapidity distribution, resummation similar to the inclusive one, with a single scaling variable, can be obtained in certain kinematic regions, see [44-48] and an equivalent approach based on SCET can be found in $[49,50]$. In the former one, called the standard direct QCD (dQCD) approach [1,33,34], since the resummation is performed in Mellin space where the phase space of the soft gluons factorizes under appropriate Mellin transformation, the threshold limit of the partonic scaling variable $z \rightarrow 1$ corresponds to Mellin variable $N \rightarrow \infty$, where $z=q^{2} / \hat{s}, q^{2}=M_{V}, V=Z, W^{ \pm}$ and $\hat{s}$ is the partonic center of mass energy. In SCET approach $[41,49,50]$, however, resummation can be performed both in Mellin space as well as in $z$-space using the evolution operators of soft and the hard functions of the coefficient function.

Resummation of large logarithms for rapidity distribution has been an interesting topic and several results are already available to very good accuracy. In [46] the authors have studied resummation of rapidity $W^{ \pm}$in Mellin-Fourier (M-F) space following a conjecture (see [44]) and later on same approach was used for Drell-Yan production in [47]. A more detailed study in the context of $W^{ \pm}$productions as well as production of a pair of leptons was undertaken in [51] emphasizing the role of prescriptions that take care of diverging series at a given logarithmic accuracy.

In this article we will follow the dQCD approach [1] to study soft gluon resummation for the rapidity distribution of a pair of leptons produced in hadron colliders. Recently, using the formalism developed in $[2,19]$, we [3] derived a general result, applicable to production of any colorless state in hadron colliders, that resums the soft gluons to all orders in perturbation theory in two dimensional Mellin (M-M) space spanned by $N_{1}, N_{2}$. We also investigated their numerical impact on the rapidity distribution of the Higgs boson produced at the LHC. The soft gluon effects show up through delta functions and plus distributions in the partonic cross sections when the partonic scaling variables reach the threshold limits, i.e., $z_{1} \rightarrow 1$ and $z_{2} \rightarrow 1$ and these contributions can be resummed to all orders both in $z_{1}, z_{2}$ space and in $N_{1}, N_{2}$ space. These resummed results were expanded to the desired accuracy to obtain fixed order predictions for various observables $[19,20,52]$ at the LHC in the SV approximation. This double threshold limit, denoted by a pair of limits, namely $\left(z_{1} \rightarrow 1, z_{2} \rightarrow 1\right)$ corresponds to $\left(N_{1} \rightarrow \infty, N_{2} \rightarrow \infty\right)$ in M-M space. The corresponding large logarithms are of the form $\ln ^{n}\left(N_{i}\right)$, where $n=1, \ldots$ and $i=1,2$ and the resummation in M-M space resums terms of the form $\omega=a_{s} \beta_{0} \ln \left(N_{1} N_{2}\right)$ through a process independent function $g(\omega)$ and a process dependent but $N_{i}$ independent function $g_{0}$. Here $\beta_{0}$ is the leading coefficient of the beta function of the strong coupling constant $g_{s}$ and $a_{s}=g_{s}^{2}\left(\mu_{R}^{2}\right) / 16 \pi^{2}$ with $\mu_{R}$ being the renormalization scale.

The main goal of this article is to study the numerical impact of resummed contributions in the M-M approach on the fixed order predictions for the rapidity distribution of a pair of leptons in the Drell-Yan process at the LHC. At NNLO level, fixed order results show remarkable stability against the factorization and renormalization scales. In addition, they demonstrate excellent perturbative 
convergence. While this is a good news for any phenomenological study with Drell-Yan process, the question remains whether the fixed order predictions will be plagued by presence of large kinematic logarithms resulting from soft gluons in the threshold regions at every order in perturbative expansion. The formalisms that can resum these large logarithms to all orders do exist and it is not a priori clear whether the resulting resummed contributions will not affect the fixed order predictions. Hence, a detailed study taking into account these threshold effects through resummation is warranted. In addition, owing to various ways by which these logarithms can be resummed, a detailed comparison of these approaches is desirable. This article attempts to address all these issues. We start by recapitulating the resummation formalism based on M-M approach and then proceed with a detailed numerical study at the LHC and conclude with our findings.

\section{THEORETICAL FRAMEWORK}

In the QCD improved parton model, for the production of a pair of leptons with invariant mass $q^{2}$ and rapidity $y$, the double differential cross section can be written as

$$
\begin{aligned}
\frac{d^{2} \sigma^{q}\left(\tau, q^{2}, y\right)}{d q^{2} d y}= & \sigma_{\mathrm{B}}^{q}\left(x_{1}^{0}, x_{2}^{0}, q^{2}\right) \sum_{a b=q, \bar{q}} \int_{x_{1}^{0}}^{1} \frac{d z_{1}}{z_{1}} \int_{x_{2}^{0}}^{1} \frac{d z_{2}}{z_{2}} \\
& \times f_{a}\left(\frac{x_{1}^{0}}{z_{1}}, \mu_{F}^{2}\right) f_{b}\left(\frac{x_{2}^{0}}{z_{2}}, \mu_{F}^{2}\right) \\
& \times \Delta_{d, a b}^{q}\left(z_{1}, z_{2}, q^{2}, \mu_{F}^{2}, \mu_{R}^{2}\right),
\end{aligned}
$$

where $\sigma_{\mathrm{B}}^{q}\left(x_{1}^{0}, x_{2}^{0}, q^{2}\right)$ is the Born prefactor, $\tau=q^{2} / S=$ $x_{1}^{0} x_{2}^{0}$ with $q$ being the momentum of the final state lepton pairs and $S=\left(p_{1}+p_{2}\right)^{2}$ where $p_{i}$ are the momenta of the incoming hadrons. The hadronic rapidity is defined as $y=\frac{1}{2} \ln \left(\frac{p_{2} \cdot q}{p_{1} \cdot q}\right)=\frac{1}{2} \ln \left(\frac{x_{1}^{0}}{x_{2}^{0}}\right) ; f_{a}\left(\frac{x_{1}^{0}}{z_{1}}, \mu_{F}^{2}\right)$ and $f_{b}\left(\frac{x_{2}^{0}}{z_{2}}, \mu_{F}^{2}\right)$ are the PDFs having momentum fractions $x_{1}=x_{1}^{0} / z_{1}, x_{2}=x_{2}^{0} / z_{2}$ respectively, renormalized at the factorization scale $\mu_{F}$. $\Delta_{d, a b}^{q}\left(a_{s}, z_{1}, z_{2}, q^{2}, \mu_{F}^{2}\right)$ [shorthand as $\Delta_{d, a b}^{q}\left(z_{1}, z_{2}\right)$ ] on the other hand is the Drell-Yan coefficient function for the rapidity distribution mass factorized at $\mu_{F}$. Unlike PDFs, these are calculable order by order in QCD perturbation theory in powers of $a_{s}$. The coefficients in this power series expansion contain distributions such as $\delta\left(1-z_{i}\right)$ and $\left[\frac{\ln ^{m-1}\left(1-z_{i}\right)}{1-z_{i}}\right]_{+}$with $m \leq 2 n, n$ being the order of perturbation and regular functions of $z_{i}$. The former ones, namely the distributions, constitute the SV part, denoted by $\Delta_{d, a b}^{q, \mathrm{SV}}$ while the latter one, the hard part is $\Delta_{d, a b}^{q, H}$. In the SV part, these distributions result from certain regions of the phase space in the real emission sub processes and also of the loop integrals in the virtual ones. While these distributions are singular as $z_{i} \rightarrow 1$, they are integrable functions. At the level of hadronic cross sections, they often dominate over the hard part when folded with the appropriate PDFs, in the above mentioned kinematic regions at every order in perturbation theory. Hence, they can potentially disturb the reliability of the perturbative predictions. The resolution is to resum these large terms, often the logarithms, to all orders to obtain any sensible prediction. This is indeed the case for the rapidity distribution in Drell-Yan when the scaling variables $z_{1} \rightarrow 1$ and $z_{2} \rightarrow 1$. Recall that in the work by Catani and Trentadue [1], a different distribution namely Feynman $x_{F}$ for the Drell-Yan was studied in the context of threshold resummation and it was shown that the potential threshold logarithms can be resummed to all orders in perturbation theory working in M-M space. They had established that these logarithms could be exponentiated and also obtained the resummed result at the next-toleading-logarithmic (NLL) accuracy. Following this, in $[2,19,20]$ we demonstrated for the rapidity distribution of any colorless particle, resummation of the distributions defined with respect to the scaling variables $z_{i}$ to all orders in perturbation theory in $z_{1}, z_{2}$ space and later extended it to $N_{1}, N_{2}$ space in [3] by applying two dimensional Mellin transformations on these distributions to obtain resummed result in the M-M space. The latter one, namely the resummed result in the M-M space, turned out to be more suitable for numerical study and hence, we used this approach to demonstrate the importance of these threshold logarithms for the rapidity distribution of the Higgs boson at the hadron collider to next-to-next-to-leading-logarithmic (NNLL) accuracy [3] over NNLO. In this paper, we explore this approach to study the Drell-Yan process at the LHC. We use the general result obtained for any colorless particle in [3] to study the numerical impact on the rapidity distribution of a pair of leptons produced at the LHC.

Note that the approach followed here [3] differs from earlier ones (see $[44,45,47,49-51]$ in the way the threshold limit(s) is(are) taken). In the latter approach, the threshold contributions from soft gluons in the partonic cross section are defined by considering only those distributions with respect to the scaling variable $z=z_{1} z_{2}$ which appear in the region when $z \rightarrow 1$. The remaining contributions contain not only regular terms in $z$ but also distributions and regular functions of partonic rapidity variable $\left(y_{p}\right)$. Here, only distributions in $z$ are resummed to all orders treating the remaining terms as hard part. Thus the resummation for the Drell-Yan rapidity distributions has been done using a single Mellin variable $N$ corresponding to $z$ and keeping the $y_{p}$ dependent coefficients as it is. Interestingly, if one works in M-F space, it can be easily shown that in the limit $z \rightarrow 1$, the threshold logarithms resulting from $N \rightarrow \infty$ are identical to those of the inclusive cross section. Unlike the M-F approach where contributions resulting from $y_{p} \neq 0$ are dropped in the resummed formula, the methodology demonstrated in the present paper includes the threshold logarithms coming from $y_{p} \neq 0$ region as well, hence covering wide range of values for the variable $y_{p}$. The advantage of our approach is in constraining the PDFs at high momentum 
fraction. In particular, dilepton pair at large $y$ resulting from collisions in which one of the partons carries a large and the other a small momentum fraction $x$, can be used to constrain the PDFs at large $x$, a region not well constrained by the current results.

We employ the technique developed in [3] namely the M-M space approach to perform the soft gluon resummation for Drell-Yan rapidity distribution. Thanks to the convolution structure of the hadronic cross section in terms of the PDFs $f_{a, b}$ and the Drell-Yan coefficient functions $\Delta_{d, a b}^{q}$, the two-dimensional Mellin transformation of the Born normalized hadronic cross section becomes a simple product of $\tilde{f}_{a}\left(N_{1}\right), \tilde{f}_{b}\left(N_{2}\right)$ and $\tilde{\Delta}_{d, a b}^{q}\left(N_{1}, N_{2}\right)$ where $\tilde{f}_{c}\left(N_{i}\right)=\int_{0}^{1} d z_{i} z_{i}^{N_{i}-1} f_{c}\left(z_{i}\right)$ for $i=1,2, c=a, b$ and

$$
\tilde{\Delta}_{d, a b}^{q}\left(N_{1}, N_{2}\right)=\left[\prod_{i=1,2} \int_{0}^{1} d z_{i} z_{i}^{N_{i}-1}\right] \Delta_{d, a b}^{q}\left(z_{1}, z_{2}\right)
$$

It was shown in $[2,3,19]$ that the SV part of $\tilde{\Delta}_{d, a b}^{q}\left(\tilde{\Delta}_{d, q}^{\mathrm{SV}}\right)$ exponentiates the threshold logarithms through the cusp anomalous dimension $A^{q}$ and the collinear functions $D_{d}^{q}$ giving the resummed result

$$
\begin{aligned}
& \tilde{\Delta}_{d, q}^{\mathrm{SV}}\left(N_{1}, N_{2}\right) \\
& =g_{d, 0}^{q}\left(a_{s}\right) \exp \left(\left[\prod_{i=1,2} \int d z_{i} z_{i}^{N_{i}-1}\right]\right. \\
& \quad \times\left[\delta\left(\bar{z}_{2}\right)\left(\frac{1}{\bar{z}_{1}}\left\{\int_{\mu_{F}^{2}}^{q^{2} \bar{z}_{1}} \frac{d \lambda^{2}}{\lambda^{2}} A^{q}\left(a_{s}\left(\lambda^{2}\right)\right)+D_{d}^{q}\left(a_{s}\left(q^{2} \bar{z}_{1}\right)\right)\right\}\right)_{+}\right. \\
& +\frac{1}{2}\left(\frac{1}{\bar{z}_{1} \bar{z}_{2}}\left\{A^{q}\left(a_{s}\left(q^{2} \bar{z}_{1} \bar{z}_{2}\right)\right)+\frac{d D_{d}^{q}\left(a_{s}\left(q^{2} \bar{z}_{1} \bar{z}_{2}\right)\right)}{d \ln \left(q^{2} \bar{z}_{1} \bar{z}_{2}\right)}\right\}\right)_{+} \\
& \left.\left.+\left(z_{1} \leftrightarrow z_{2}\right)\right]\right),
\end{aligned}
$$

where $\bar{z}_{i}=\left(1-z_{i}\right)$. The cusp anomalous dimensions for the quark, $A^{q}$, are known for Drell-Yan up to 3-loops [53-58], and the coefficients in $A^{q}=\sum_{i=1}^{\infty} a_{s}^{i} A_{i}^{q}$ are given by

$$
\begin{aligned}
A_{1}^{q}= & 4 C_{F}, \\
A_{2}^{q}= & 8 C_{F} C_{A}\left(\frac{67}{18}-\zeta_{2}\right)+8 C_{F} n_{f}\left(-\frac{5}{9}\right), \\
A_{3}^{q}= & 16 C_{F} C_{A}^{2}\left(\frac{245}{24}-\frac{67}{9} \zeta_{2}+\frac{11}{6} \zeta_{3}+\frac{11}{5} \zeta_{2}^{2}\right) \\
& +16 C_{F}^{2} n_{f}\left(-\frac{55}{24}+2 \zeta_{3}\right)+16 C_{F} C_{A} n_{f} \\
& \times\left(-\frac{209}{108}+\frac{10}{9} \zeta_{2}-\frac{7}{3} \zeta_{3}\right)-16 C_{F} n_{f}^{2}\left(\frac{1}{27}\right),
\end{aligned}
$$

and $D_{d}^{q} \mathrm{~s}$ are related $[2,3,19]$ to the $D^{q} \mathrm{~S}$ of the inclusive cross section for the Drell-Yan. Expanding $D_{d}^{q}$ as $D_{d}^{q}=$ $\sum_{i=1}^{\infty} a_{s}^{i} D_{d, i}^{q}$, we find that $D_{d, 1}^{q}=0, \quad D_{d, 2}^{q}=C_{f} n_{f}$ $\left\{\frac{112}{27}-\frac{8}{3} \zeta_{2}\right\}+C_{a} C_{f}\left\{-\frac{808}{27}+28 \zeta_{3}+\frac{44}{3} \zeta_{2}\right\}$, with the SU(N) color factors

$$
C_{A}=\mathrm{N}, \quad C_{F}=\frac{\mathrm{N}^{2}-1}{2 \mathrm{~N}}, \quad T_{F}=\frac{1}{2}
$$

and $n_{f}$ is the number of active flavors. In [3], following [59], we systematically computed the two dimensional Mellin transformations in the large $N_{i}$ limits and the result takes the following form:

$$
\tilde{\Delta}_{d, q}^{\mathrm{SV}}\left(N_{1}, N_{2}\right)=\tilde{g}_{d, 0}^{q}\left(a_{s}\right) \exp \left(g_{d}^{q}\left(a_{s}, \omega\right)\right),
$$

where $\omega$ is defined as $\omega=a_{s} \beta_{0} \ln \left(\bar{N}_{1} \bar{N}_{2}\right)$, with $\bar{N}_{i}=e^{\gamma_{E}} N_{i}$, $i=1,2$. The coefficients $g_{d}\left(a_{s}, \omega\right)$ are process independent and contain purely logarithmically enhanced terms and can be expanded as,

$$
g_{d}^{q}\left(a_{s}, \omega\right)=g_{d, 1}^{q}(\omega) \ln \left(\bar{N}_{1} \bar{N}_{2}\right)+\sum_{i=0}^{\infty} a_{s}^{i} g_{d, i+2}^{q}(\omega) .
$$

Rescaling the constants by appropriate $\beta_{i}$ as $\bar{g}_{d, 1}^{q}=g_{d, 1}^{q}$, $\bar{g}_{d, 2}^{q}=g_{d, 2}^{q}, \quad \bar{g}_{d, 3}^{q}=g_{d, 3}^{q} / \beta_{0}, \quad \bar{A}_{i}^{q}=A_{i}^{q} / \beta_{0}^{i}, \quad \bar{D}_{d, i}^{q}=D_{d, i}^{q} / \beta_{0}^{i}$ and $\bar{\beta}_{i}=\beta_{i} / \beta_{0}^{i+1}$, we find [3].

$$
\begin{aligned}
\bar{g}_{d, 1}^{q}= & \bar{A}_{1}^{I} \frac{1}{\omega}\{\omega+(1-\omega) \ln (1-\omega)\}, \\
\bar{g}_{d, 2}^{q}= & \omega\left\{\bar{A}_{1}^{I} \bar{\beta}_{1}-\bar{A}_{2}^{I}\right\}+\ln (1-\omega)\left\{\bar{A}_{1}^{I} \bar{\beta}_{1}+\bar{D}_{d, 1}^{I}-\bar{A}_{2}^{I}\right\}+\frac{1}{2} \ln ^{2}(1-\omega) \bar{A}_{1}^{I} \bar{\beta}_{1}+\ln \left(\frac{q^{2}}{\mu_{R}^{2}}\right) \ln (1-\omega) \bar{A}_{1}^{I}+\ln \left(\frac{\mu_{F}^{2}}{\mu_{R}^{2}}\right) \omega \bar{A}_{1}^{I}, \\
\bar{g}_{d, 3}^{q}= & -\frac{1}{2} \omega \bar{A}_{3}^{I}-\frac{1}{2} \frac{\omega}{1-\omega}\left\{-\bar{A}_{3}^{I}+(2+\omega) \bar{\beta}_{1} \bar{A}_{2}^{I}+\left\{(w-2) \bar{\beta}_{2}-\omega \bar{\beta}_{1}^{2}-2 \zeta_{2}\right\} \bar{A}_{1}^{I}+2 \bar{D}_{d, 2}^{I}-2 \bar{\beta}_{1} \bar{D}_{d, 1}^{I}\right\} \\
& -\ln (1-\omega)\left\{\frac{\bar{\beta}_{1}}{1-\omega}\left\{\bar{A}_{2}^{I}-\bar{D}_{d, 1}^{I}-\bar{A}_{1}^{I} \bar{\beta}_{1} \omega\right\}-\bar{A}_{1}^{I} \bar{\beta}_{2}\right\}+\frac{1}{2} \frac{\ln ^{2}(1-\omega)}{1-\omega} \bar{A}_{1}^{I} \bar{\beta}_{1}^{2}+\ln \left(\frac{\mu_{F}^{2}}{\mu_{R}^{2}}\right) \bar{A}_{2}^{I} \omega \\
& -\frac{1}{2} \ln ^{2}\left(\frac{\mu_{F}^{2}}{\mu_{R}^{2}}\right) \bar{A}_{1}^{I} \omega-\ln \left(\frac{q^{2}}{\mu_{R}^{2}}\right) \frac{1}{1-\omega}\left\{\left\{\bar{A}_{2}^{I}-\bar{D}_{d, 1}^{I}\right\} \omega-\bar{A}_{1}^{I} \bar{\beta}_{1}\{\omega+\ln (1-\omega)\}\right\}+\frac{1}{2} \ln ^{2}\left(\frac{q^{2}}{\mu_{R}^{2}}\right) \frac{\omega}{1-\omega} \bar{A}_{1}^{I} .
\end{aligned}
$$

The first three coefficients of the QCD $\beta$ function, $\beta_{0}, \beta_{1}$, and $\beta_{2}$ are given by [60] 


$$
\begin{aligned}
& \beta_{0}=\frac{11}{3} C_{A}-\frac{4}{3} T_{F} n_{f}, \\
& \beta_{1}=\frac{34}{3} C_{A}^{2}-4 T_{F} n_{f} C_{F}-\frac{20}{3} T_{F} n_{f} C_{A}, \\
& \beta_{2}=\frac{2857}{54} C_{A}^{3}-\frac{1415}{27} C_{A}^{2} T_{F} n_{f}+\frac{158}{27} C_{A} T_{F}^{2} n_{f}^{2}+\frac{44}{9} C_{F} T_{F}^{2} n_{f}^{2}-\frac{205}{9} C_{F} C_{A} T_{F} n_{f}+2 C_{F}^{2} T_{F} n_{f} .
\end{aligned}
$$

The $N_{1}, N_{2}$ independent terms resulting from integrals have been absorbed in $\tilde{g}_{d, 0}^{q}$ addition to $g_{d, 0}^{q}$, which however depends on the specific process under study. In principle, these $N_{1}, N_{2}$ independent terms can also be exponentiated. For Drell-Yan production, expanding $\tilde{g}_{d, 0}^{q}=\sum_{i=0}^{\infty} a_{s}^{i} \tilde{g}_{d, 0}^{q(i)}$, we find up to $a_{s}^{2}$ order in strong coupling:

$$
\begin{aligned}
\tilde{g}_{d, 0}^{q(0)}= & 1, \\
\tilde{g}_{d, 0}^{q(1)}= & C_{F}\left\{-16+16 \zeta_{2}\right\}+\ln \left(\frac{\mu_{F}^{2}}{\mu_{R}^{2}}\right) C_{F}\{-6\}+\ln \left(\frac{q^{2}}{\mu_{R}^{2}}\right) C_{F}\{6\}, \\
\tilde{g}_{d, 0}^{q(2)}= & C_{F} n_{f}\left\{\frac{127}{6}-\frac{64}{3} \zeta_{2}+\frac{8}{9} \zeta_{3}\right\}+C_{F}^{2}\left\{\frac{511}{4}-198 \zeta_{2}-60 \zeta_{3}+\frac{552}{5} \zeta_{2}^{2}\right\}+C_{A} C_{F}\left\{-\frac{1535}{12}+\frac{376}{3} \zeta_{2}+\frac{604}{9} \zeta_{3}-\frac{92}{5} \zeta_{2}^{2}\right\} \\
& +\ln \left(\frac{\mu_{F}^{2}}{\mu_{R}^{2}}\right)\left[C_{F} n_{f}\left\{\frac{2}{3}+\frac{16}{3} \zeta_{2}\right\}+C_{F}^{2}\left\{93-72 \zeta_{2}-48 \zeta_{3}\right\}+C_{A} C_{F}\left\{-\frac{17}{3}-\frac{88}{3} \zeta_{2}+24 \zeta_{3}\right\}\right] \\
& +\ln ^{2}\left(\frac{\mu_{F}^{2}}{\mu_{R}^{2}}\right)\left[C_{F} n_{f}\{-2\}+C_{F}^{2}\{18\}+C_{A} C_{F}\{11\}\right]+\ln \left(\frac{q^{2}}{\mu_{R}^{2}}\right)\left[C_{F} n_{f}\left\{-\frac{34}{3}+\frac{16}{3} \zeta_{2}\right\}\right. \\
& \left.+C_{F}^{2}\left\{-93+72 \zeta_{2}+48 \zeta_{3}\right\}+C_{A} C_{F}\left\{\frac{193}{3}-\frac{88}{3} \zeta_{2}-24 \zeta_{3}\right\}\right]+\ln \left(\frac{q^{2}}{\mu_{R}^{2}}\right) \ln \left(\frac{\mu_{F}^{2}}{\mu_{R}^{2}}\right) C_{F}^{2}\{-36\} \\
& +\ln ^{2}\left(\frac{q^{2}}{\mu_{R}^{2}}\right)\left[C_{F} n_{f}\{2\}+C_{F}^{2}\{18\}+C_{A} C_{F}\{-11\}\right] .
\end{aligned}
$$

To study the numerical impact of our resummed result, we require in addition fixed order results containing only the large logarithms to perform proper matching. They can be obtained by truncating the resummed result and in the following, we present the $\tilde{\Delta}_{d, q}^{\mathrm{SV}(i)}$ by setting $\mu_{R}^{2}=\mu_{F}^{2}$ up to NNLO level:

$$
\begin{aligned}
\tilde{\Delta}_{d, q}^{\mathrm{SV}(0)}= & 1 \\
\tilde{\Delta}_{d, q}^{\mathrm{SV}(1)}= & C_{F}\left\{-16+16 \zeta_{2}\right\}+\ln ^{2}(\bar{\omega}) C_{F}\{2\}+\ln \left(\frac{q^{2}}{\mu_{F}^{2}}\right) C_{F}\{6\}+\ln \left(\frac{q^{2}}{\mu_{F}^{2}}\right) \ln (\bar{\omega}) C_{F}\{-4\} \\
\tilde{\Delta}_{d, q}^{\mathrm{SV}(2)}= & C_{F} n_{f}\left\{\frac{127}{6}-\frac{64}{3} \zeta_{2}+\frac{8}{9} \zeta_{3}\right\}+C_{F}^{2}\left\{\frac{511}{4}-198 \zeta_{2}-60 \zeta_{3}+\frac{552}{5} \zeta_{2}^{2}\right\} \\
& +C_{A} C_{F}\left\{-\frac{1535}{12}+\frac{376}{3} \zeta_{2}+\frac{604}{9} \zeta_{3}-\frac{92}{5} \zeta_{2}^{2}\right\}+\ln (\bar{\omega})\left[C_{F} n_{f}\left\{-\frac{112}{27}\right\}+C_{A} C_{F}\left\{\frac{808}{27}-28 \zeta_{3}\right\}\right] \\
& +\ln ^{2}(\bar{\omega})\left[C_{F} n_{f}\left\{-\frac{20}{9}\right\}+C_{F}^{2}\left\{-32+32 \zeta_{2}\right\}+C_{A} C_{F}\left\{\frac{134}{9}-4 \zeta_{2}\right\}\right] \\
& +\ln ^{3}(\bar{\omega})\left[C_{F} n_{f}\left\{-\frac{4}{9}\right\}+C_{A} C_{F}\left\{\frac{22}{9}\right\}\right]+\ln ^{4}(\bar{\omega}) C_{F}^{2}\{2\}+\ln \left(\frac{q^{2}}{\mu_{F}^{2}}\right) \\
& \times\left[C_{F} n_{f}\left\{-\frac{34}{3}+\frac{16}{3} \zeta_{2}\right\}+C_{F}^{2}\left\{-93+72 \zeta_{2}+48 \zeta_{3}\right\}+C_{A} C_{F}\left\{\frac{193}{3}-\frac{88}{3} \zeta_{2}-24 \zeta_{3}\right\}\right] \\
& +\ln \left(\frac{q^{2}}{\mu_{F}^{2}}\right) \ln (\bar{\omega})\left[C_{F} n_{f}\left\{\frac{40}{9}\right\}+C_{F}^{2}\left\{64-64 \zeta_{2}\right\}+C_{A} C_{F}\left\{-\frac{268}{9}+8 \zeta_{2}\right\}\right]
\end{aligned}
$$




$$
\begin{aligned}
& +\ln \left(\frac{q^{2}}{\mu_{F}^{2}}\right) \ln ^{2}(\bar{\omega})\left[C_{F} n_{f}\left\{\frac{4}{3}\right\}+C_{F}^{2}\{12\}+C_{A} C_{F}\left\{-\frac{22}{3}\right\}\right]+\ln \left(\frac{q^{2}}{\mu_{F}^{2}}\right) \ln ^{3}(\bar{\omega}) C_{F}^{2}\{-8\} \\
& +\ln ^{2}\left(\frac{q^{2}}{\mu_{F}^{2}}\right)\left[C_{F} n_{f}\{2\}+C_{F}^{2}\{18\}+C_{A} C_{F}\{-11\}\right]+\ln ^{2}\left(\frac{q^{2}}{\mu_{F}^{2}}\right) \\
& \times \ln (\bar{\omega})\left[C_{F} n_{f}\left\{-\frac{4}{3}\right\}+C_{F}^{2}\{-24\}+C_{A} C_{F}\left\{\frac{22}{3}\right\}\right]+\ln ^{2}\left(\frac{q^{2}}{\mu_{F}^{2}}\right) \ln ^{2}(\bar{\omega}) C_{F}^{2}\{8\} .
\end{aligned}
$$

where $\bar{\omega}=\bar{N}_{1} \bar{N}_{2}$. In the following, we will discuss how these resummed contributions can be systematically included in order to study their phenomenological importance at the LHC.

\section{PHENOMENOLOGY}

Our next task is to include the resummed contributions consistently in the fixed order predictions and study their numerical impact on the rapidity distribution of lepton pairs produced in the Drell-Yan process at the LHC. We consider the production of both leptons, i.e., $\ell^{+} \ell^{-}$, where $\ell=e, \mu$ through $Z$ and $\gamma^{*}$ in the collision of two hadrons at the center of mass energy $14 \mathrm{TeV}$. Unless otherwise stated, we will mostly focus on the region containing the $Z$-pole. We take $n_{f}=5$ flavors, the MMHT2014(68cl) PDF set [61] and the corresponding $a_{s}\left(M_{Z}\right)$ through the LHAPDF-6 [62] interface at each order in perturbation theory. For the fixed order rapidity distribution, we use the publicly available code Vrap-0.9 [14,63]. The resummed contribution is obtained from $\tilde{\Delta}_{d, q}^{\mathrm{SV}}\left(N_{1}, N_{2}\right)$ in Eq. (6) after performing Mellin inversions which are done using an in house Fortran based code. Since the resummed result cannot be simply added to the fixed order one because all the $\ln \left(N_{i}\right)$ and $N_{i}$ independent terms present in the resummed exponential $g_{d}^{q}$ and $\tilde{g}_{d, 0}^{q}$ are already present in the fixed order results and hence care is needed to avoid double counting. This can be achieved simply by employing a matching procedure at every order. The matched result is given below

$$
\begin{aligned}
\frac{d^{2} \sigma^{q, \text { res }}}{d q^{2} d y}= & \frac{d^{2} \sigma^{q, \text { f.o }}}{d q^{2} d y}+\sigma_{B}^{q} \int_{c_{1}-i \infty}^{c_{1}+i \infty} \frac{d N_{1}}{2 \pi i} \int_{c_{2}-i \infty}^{c_{2}+i \infty} \\
& \times \frac{d N_{2}}{2 \pi i} e^{y\left(N_{2}-N_{1}\right)}(\sqrt{\tau})^{2-N_{1}-N_{2}} \tilde{f}_{q}\left(N_{1}\right) \tilde{f}_{q}\left(N_{2}\right) \\
& \times\left[\tilde{\Delta}_{d, q}^{\mathrm{SV}}-\left.\tilde{\Delta}_{d, q}^{\mathrm{SV}}\right|_{\text {trunc }}\right],
\end{aligned}
$$

where $\sigma_{B}^{q}$ is given by

$$
\begin{aligned}
\sigma_{B}^{q}= & \frac{4 \pi \alpha^{2}}{3 q^{4} N}\left[e_{q}^{2}-\frac{2 q^{2}\left(q^{2}-M_{Z}^{2}\right) e_{q} g_{e}^{V} g_{q}^{V}}{\left(\left(q^{2}-M_{Z}^{2}\right)^{2}+M_{Z}^{2} \Gamma_{Z}^{2}\right) c_{w}^{2} s_{w}^{2}}\right. \\
& +\frac{3 q^{4} \Gamma_{Z} B_{l}^{Z}}{16 \alpha M_{Z}\left(\left(q^{2}-M_{Z}^{2}\right)^{2}+M_{Z}^{2} \Gamma_{Z}^{2}\right) c_{w}^{2} s_{w}^{2}} \\
& \left.\times\left(1+\left(1-\frac{8}{3} s_{w}^{2}\right)^{2}\right)\right]
\end{aligned}
$$

with $\alpha=\alpha\left(M_{Z}\right)=1 / 127.925, e_{q}$ is the quark charge, $M_{Z}=91.1876 \mathrm{GeV}, \Gamma_{Z}=2.4952 \mathrm{GeV}, s_{w}^{2}=0.227, c_{w}^{2}=$ $1-s_{w}^{2}, g_{e}^{V}=-1 / 4+s_{w}^{2}, g_{u}^{V}=1 / 4-2 / 3 s_{w}^{2}, g_{d}^{V}=-1 / 4+$ $1 / 3 s_{w}^{2}, B_{e}^{Z}=0.03363$ and $B_{\mu}^{Z}=0.03366$. The first term in Eq. (12), $\left(d^{2} \sigma^{q, \text { f.o }} / d q^{2} d y\right)$, corresponds to contributions resulting from a fixed order perturbative computation. The second term on the other hand contains only threshold logarithms $\ln \left(N_{i}\right)$ but to all orders in perturbation theory. The subscript "trunc" in the $\tilde{\Delta}_{d, q}^{\mathrm{SV}}$ indicates that it is truncated at the same order as the fixed order one after expanding in powers of $a_{s}$. Hence, at a given order $n$ in $a_{s}$ (i.e., at order $a_{s}^{n}$ ), the nonzero contribution from the second term starts at order $a_{s}^{n+1}$ and includes terms from all orders. For the fixed $n$th order contribution, namely $\mathrm{N}^{n} \mathrm{LO}$, the contribution from the second term is called $\mathrm{N}^{n} \mathrm{LL}$. Hence, we use the notations LO, NLO, and NNLO for the fixed order predictions and correspondingly $\mathrm{LO}+\mathrm{LL}$, NLO + NLL, and NNLO + NNLL for the resummed ones. It is well known that the resummed expression diverges due to the missing nonperturbative contributions. These divergences show up when $\omega \rightarrow 1$ in the functions $\bar{g}_{d, i}^{q}$; they are due to the coupling constant $a_{s}\left(\mu_{R}^{2}\right)$ that diverges near the Landau pole. In order to resolve this, we have adopted the minimal prescription (MP) [64]. The contours for the integrals corresponding to two Mellin inversions [65] are chosen in such a way that all the poles in the complex plane spanned by $N_{1}, N_{2}$ remain to the left of the contours except for the Landau pole. Since the leading order contribution to the Drell-Yan process is due to EW interactions, the dominant theoretical uncertainty comes from the factorization scale $\mu_{F}$ that enters through the parton distribution functions while the dependence on the renormalization scale $\mu_{R}$ starts only from NLO onwards. Unlike the leading order prediction, in the resummed case, the LL contributions do depend on $\mu_{R}$ through $\omega$ in $\bar{g}_{d, 1}^{q}(w)$ given in Eq. (8). Hence, $\mu_{R}$ dependence will show up at even LO + LL level. This will be evident from Fig. 4 that one finds larger scale uncertainty from $\mathrm{LO}+\mathrm{LL}$ contributions compared to the fixed order one at LO level. It is then important to understand the impact of these two scales at the resummed level and also to determine the optimal choice for the central scale around which the scale uncertainty remains minimal. For the fixed order case it has already been realized in [66] that the optimal choice for the central scale is when both $\mu_{R}$ and $\mu_{F}$ are set to $M_{Z}$. 


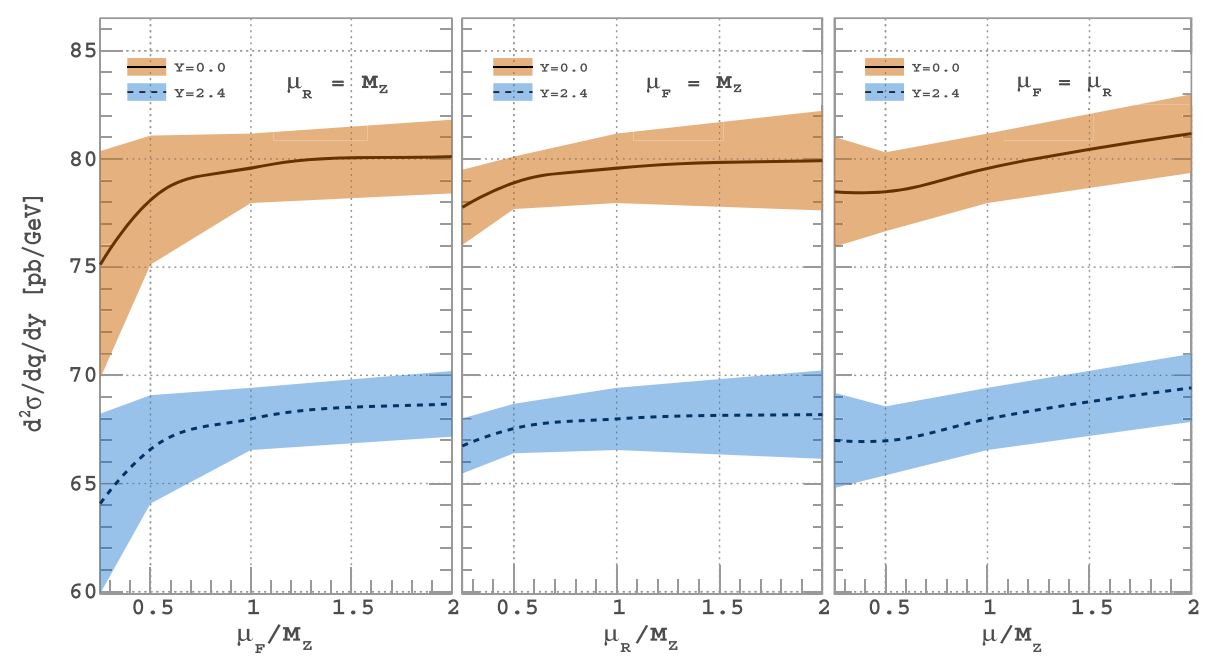

FIG. 1. Cross sections against $\mu_{F}$ (left), $\mu_{R}$ (middle) and $\mu$ (right) variations at NNLO + NNLL for 14 TeV LHC. The bands are obtained by using 7-point scale variation (see text for more details).

In order to obtain the optimized central scale for the resummed case, we have plotted in Fig. 1 the dependence of the rapidity distribution on (a) $\left(\mu_{R}=M_{Z}, \mu_{F}\right)$, (b) $\left(\mu_{R}, \mu_{F}=M_{Z}\right)$, and finally (c) $\left(\mu=\mu_{R}=\mu_{F}\right)$ at NNLO + NNLL level. The symmetric band is obtained by performing 7-point scale variation $[51,59,66]$ around a given central scale with the constraint $\left(k_{1}, k_{2}\right) \otimes\left(\mu_{R}, \mu_{F}\right)_{\text {central }}$ where $\left(k_{1}, k_{2}\right) \in[1 / 2,2]$ with $1 / 2 \leq k_{1} / k_{2} \leq 2$ and by taking maximum absolute deviation from the central scale. From the first and the last panels of Fig. 1, it is clear that the optimal central scale choice is $\left(M_{Z}, M_{Z}\right)$ whereas the middle panel favors $\left(M_{Z} / 2, M_{Z}\right)$ for the central scale. Comparing all three panels, we find that the choice $\left(M_{Z} / 2, M_{Z}\right)$ gives

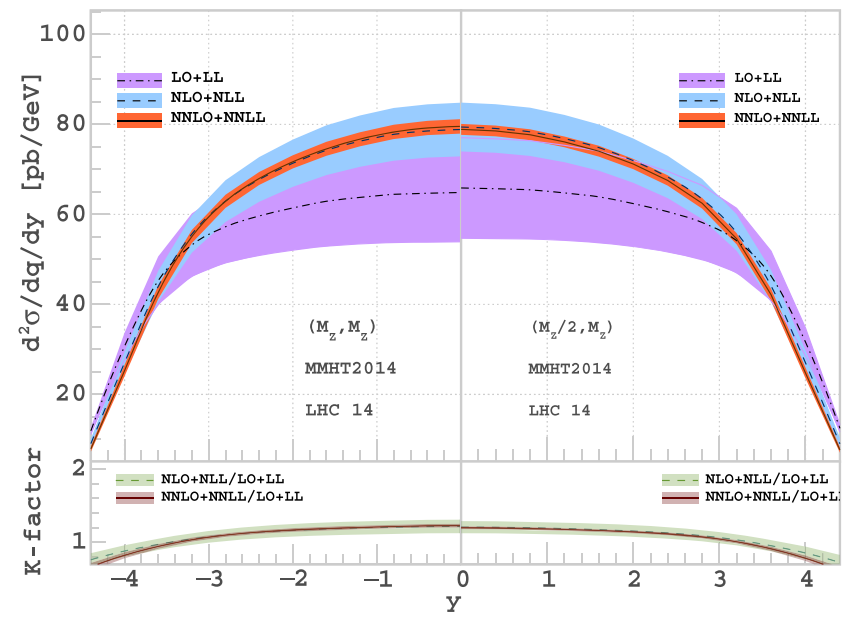

FIG. 2. Resummed rapidity distribution in Drell-Yan production for the two sets of central scale choices $\left(M_{Z}, M_{Z}\right)$ and $\left(M_{Z} / 2, M_{Z}\right)$ using MMHT PDFs at 14 TeV LHC. Corresponding bands are obtained using 7-point scale variation around the central scale. The lower panel represents the corresponding K-factors. minimum uncertainty band. However, to confirm the above analysis also holds true at each order in the perturbation theory, we have considered two different central scale choices $\left(M_{Z}, M_{Z}\right)$ and $\left(M_{Z} / 2, M_{Z}\right)$ in Fig. 2 . We find that while the acceleration of the perturbative convergence are almost same for both cases, uncertainty band at NLO + NLL and at NNLO + NNLL level are smaller for the central scale choice $\left(M_{Z} / 2, M_{Z}\right)$ compared to the case $\left(M_{Z}, M_{Z}\right)$. In Fig. 3, we compare predictions from the fixed order using the central scale $\left(M_{Z}, M_{Z}\right)$ against those from the resummed result using the central scale $\left(M_{Z} / 2, M_{Z}\right)$ for two rapidities $y=0$ and $y=2.4$. The scale uncertainties from the resummed case at NNLO + NNLL are comparable to what one obtains from NNLO. However, the central values at NLO + NLL and $\mathrm{NNLO}+\mathrm{NNLL}$ are very close to each other compared to

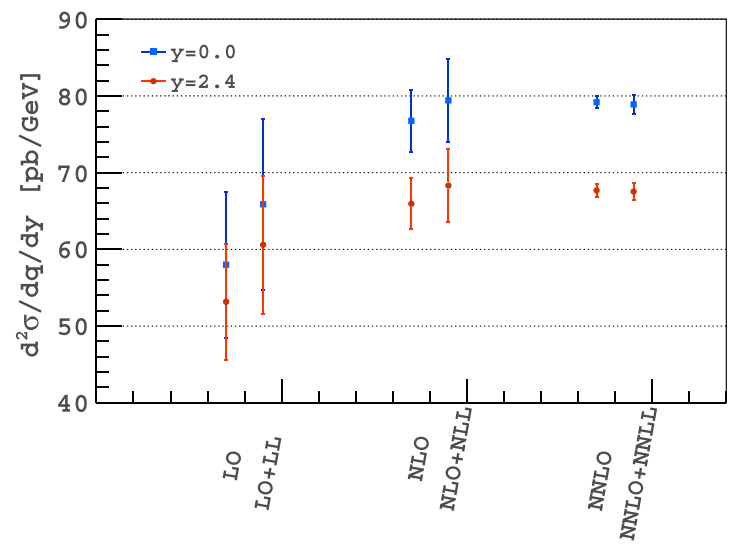

FIG. 3. Fixed order predictions with the central scale $\mu_{R}=$ $\mu_{F}=M_{Z}$ and resummed prediction with the central scale $\mu_{R}=M_{Z} / 2, \mu_{F}=M_{Z}$ for rapidity $y=0$ and $y=2.4$ using MMHT2014 PDF at each order. The uncertainties are obtained by using 7-point scale variation (see text for more details) around the central scale. 
TABLE I. Comparison of resummed results between M-F and M-M approach in the minimal prescription scheme at $y=0$ for various choices of scales.

\begin{tabular}{lcccccccccc}
\hline \hline $\mathrm{y}$ & $\left(\frac{\mu_{R}}{M_{Z}}, \frac{\mu_{F}}{M_{Z}}\right)$ & $\mathrm{LO}$ & $\mathrm{LL}_{\mathrm{M}-\mathrm{F}}$ & $\mathrm{LL}_{\mathrm{M}-\mathrm{M}}$ & $\mathrm{NLO}$ & $\mathrm{NLL}_{\mathrm{M}-\mathrm{F}}$ & $\mathrm{NLL}_{\mathrm{M}-\mathrm{M}}$ & $\mathrm{NNLO}$ & $\mathrm{NNLL}_{\mathrm{M}-\mathrm{F}}$ & NNLL $_{\mathrm{M}-\mathrm{M}}$ \\
\hline 0.0 & $(2,2)$ & 72.626 & +0.988 & +3.219 & 73.450 & +1.639 & +1.796 & 70.894 & +0.630 & +0.646 \\
0.0 & $(2,1)$ & 63.197 & +0.768 & +2.595 & 70.625 & +0.761 & +1.017 & 70.360 & +0.292 & +0.317 \\
0.0 & $(1,2)$ & 72.626 & +1.095 & +3.577 & 73.535 & +1.912 & +1.760 & 70.509 & +0.510 & +0.395 \\
0.0 & $(1,1)$ & 63.197 & +0.851 & +2.887 & 71.395 & +0.858 & +0.901 & 70.537 & +0.248 & +0.167 \\
0.0 & $(1,1 / 2)$ & 53.241 & +0.621 & +2.216 & 67.581 & +0.156 & +0.140 & 69.834 & -0.001 & -0.094 \\
0.0 & $(1 / 2,1)$ & 63.197 & +0.953 & +3.278 & 72.355 & +0.945 & +0.681 & 70.266 & +0.091 & -0.015 \\
0.0 & $(1 / 2,1 / 2)$ & 53.241 & +0.695 & +2.504 & 69.259 & +0.102 & -0.154 & 70.283 & -0.039 & -0.146 \\
\hline \hline
\end{tabular}

those of fixed order results demonstrating the better perturbative convergence. As we have discussed in the Introduction, in $[46,47,51]$ resummation of threshold logarithms for the rapidity distribution was achieved in the M-F space. Our formalism [3] differs from the other approach in the way the threshold contributions are resummed. We resum large logs resulting from the regions where scaling variables $z_{1}$ and $z_{2}$ approach unity simultaneously while in the case of M-F, only large logarithms from the region where the partonic threshold variable $z$ approaches unity and the partonic rapidity $y_{p}$ is zero, are resummed. In the following we will make the numerical comparison of our predictions, namely the M-M formalism against those of M-F reported in [51]. The fixed order contributions are obtained by using Vrap-0.9 [14,63]; the resummed contributions up to NNLL for M-F are obtained by using publicly available code ReDY [67] and for M-M, we use our in house Fortran routine. We have set all the parameters including the PDF set (NLO set of NNPDF-2.0 [68] at every order) same as those used in [51]. Both our results and those from ReDY are listed in the Table I for various scale choices at the central rapidity. At LL level, both M-F and M-M give positive contributions but the contribution from $\mathrm{M}-\mathrm{M}$ is about three times larger compared to M-F independent of the scale choice. The additional contribution over LL at NLL for M-F is negative for some scale choices and positive for the rest while for M-M, it is always negative. The magnitude of these additional contributions for $\mathrm{M}-\mathrm{M}$ is larger than M-F. Interestingly, at NNLL level, the additional contributions over NLL for M-F and M-M are both negative in a such a way that the net NNLL contributions from both approaches become comparable. In the case of M-F, the $\mathrm{NLO}+\mathrm{NLL}$ is $2 \%$ larger compared to LO + LL and NNLO + NNLL is $-4.7 \%$ larger compared NLO + NLL. For M-M, the corresponding ones are $-0.8 \%$ and $-4.9 \%$ respectively at $\mu_{R}=\mu_{F}=2 M_{Z}$.

In Fig. 4, using Eq. (12), we present the cross section for producing lepton pairs as a function of the rapidity $y$ up to NNLO in the left panel and to NNLO + NNLL in the right panel along with the respective $\mathrm{K}$-factors. The $\mathrm{K}$-factor at a given perturbative order, say at $\mathrm{N}^{n} \mathrm{LO}\left(\mathrm{N}^{n} \mathrm{LO}+\mathrm{N}^{n} \mathrm{LL}\right)$, is

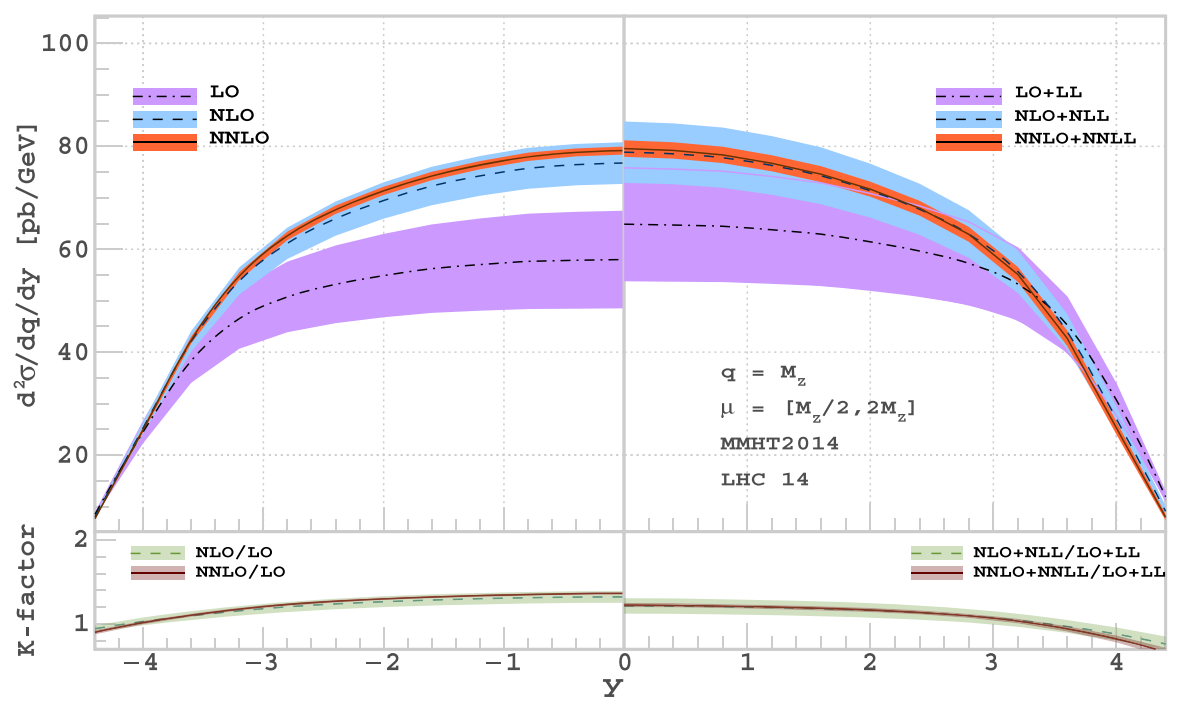

FIG. 4. Drell-Yan rapidity distribution for $14 \mathrm{TeV} \mathrm{LHC}$ at $q=M_{Z}$ using MMHT PDFs. The fixed order results are plotted in the left panel and the resummed results in the right panel. Central scale is chosen as $\mu_{R}=\mu_{F}=M_{Z}$ for both and the corresponding bands are obtained using 7-point scale variation (see text for more details) around the central scale. The lower panel represents the corresponding K-factors. 
TABLE II. Fixed order and the resummed cross sections with \% scale uncertainties along with the K-factors at the central scale $\mu_{R}=\mu_{F}=M_{Z}$.

\begin{tabular}{|c|c|c|c|c|c|c|c|c|c|c|}
\hline $\mathrm{y}$ & LO & $\mathrm{LO}+\mathrm{LL}$ & NLO & $\mathrm{NLO}+\mathrm{NLL}$ & NNLO & NNLO + NNLL & $\mathrm{K}_{\mathrm{NLO}}$ & $\mathrm{K}_{\mathrm{NLO}+\mathrm{NLL}}$ & $\mathrm{K}_{\mathrm{NNLO}}$ & $\mathrm{K}_{\mathrm{NNLO}+\mathrm{NNLL}}$ \\
\hline 0.0 & $58.002 \pm 1$ & $64.873 \pm 16.89 \%$ & $76.758 \pm 5.28 \%$ & $78.867 \pm 7.56 \%$ & $79.182 \pm 0.9$ & $79.568 \pm 2.02 \%$ & 1.323 & 1.216 & 1.365 & 1.226 \\
\hline 0.8 & $57.645 \pm 16$ & $64.468 \pm 16.61 \%$ & $75.727 \pm 5.26 \%$ & $77.797 \pm 7.53 \%$ & $77.968 \pm$ & $03 \%$ & 1.314 & 1.2 & 1.352 & 1.215 \\
\hline 1.6 & $56228+15$ & $62.929 \pm 15.82 \%$ & & & & & & & & 1.185 \\
\hline 2.4 & $19 \%$ & $59.655 \pm$ & $65.953 \pm$ & $67.772 \pm$ & $67.678 \pm 1.21 \%$ & $67.985 \pm 2.11 \%$ & 1.240 & 1.136 & 1.273 & 1.140 \\
\hline
\end{tabular}

defined by the cross section at that order normalized by the same at $\mathrm{LO}(\mathrm{LO}+\mathrm{LL})$ at the central scale $\mu_{R}=\mu_{F}=M_{Z}$. We have made this choice for the scales because the fixed order perturbative prediction is well behaved around this scale [66]. The symmetric band at each order is obtained by varying $\mu_{R}$ and $\mu_{F}$ between $\left[M_{Z} / 2,2 M_{Z}\right]$ around the central scale $\mu_{R}=\mu_{F}=M_{Z}$ with the constraint $1 / 2 \leq \mu_{R} / \mu_{F} \leq 2$, by adding and subtracting to the central scale the highest

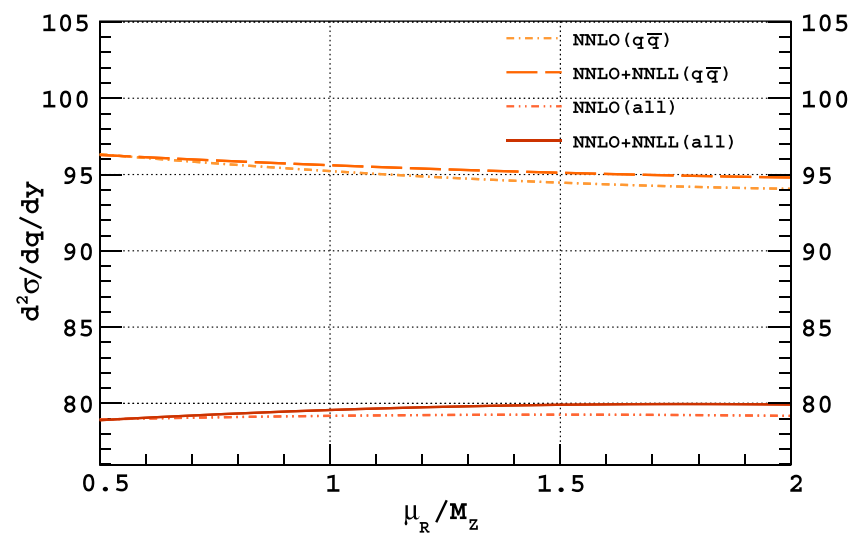

FIG. 5. Drell-Yan rapidity distribution for $14 \mathrm{TeV}$ LHC at $y=$ 0 using MMHT PDFs. The variation of fixed order and resummed results as a function of $\mu_{R}$ are shown separately for $q \bar{q}$ channel and also for all the channels added together. possible uncertainties originating from all the scale combinations. We find that the magnitude and the sign of the resummed contribution are sensitive to the order of perturbation as well the exact values of $y$ and the scales $\mu_{R}, \mu_{F}$. For example, if we choose $\mu_{R}=M_{Z} / 2$ and $\mu_{F}=M_{Z}$ instead of $\mu_{R}=\mu_{F}=M_{Z}$ as the central scale, we obtain a negative contribution from NNLL terms for all values of rapidity.

Figure 4 also demonstrates that the inclusion of $N^{n} \mathrm{LL}$ contributions increase the cross section at every order for a wide range of rapidity values. In addition, the overlap among various orders is larger for the resummed case compared to the fixed order ones, because the uncertainty band at each order in the resummed case is bigger compared to fixed order. As far as fixed order results are concerned, in particular at NNLO level, several partonic channels open up, effectively reducing the scale uncertainty considerably. On the other hand, resummed contributions come only from quark antiquark initiated channels to all orders in perturbation theory as other channels do not give threshold logarithms of the type that is resummed. We confirm this through Fig. 5, where we have studied the effects of resummation over the fixed order contributions, by considering (a) only $q \bar{q}$ channel at NNLO and (b) all the channels at NNLO. We perform our analysis for $y=0$ and set $\mu_{F}=M_{Z}$ while varying $\mu_{R}$ between $M_{Z} / 2$ to $2 M_{Z}$. For the $q \bar{q}$ channel the resum contributions arising from the

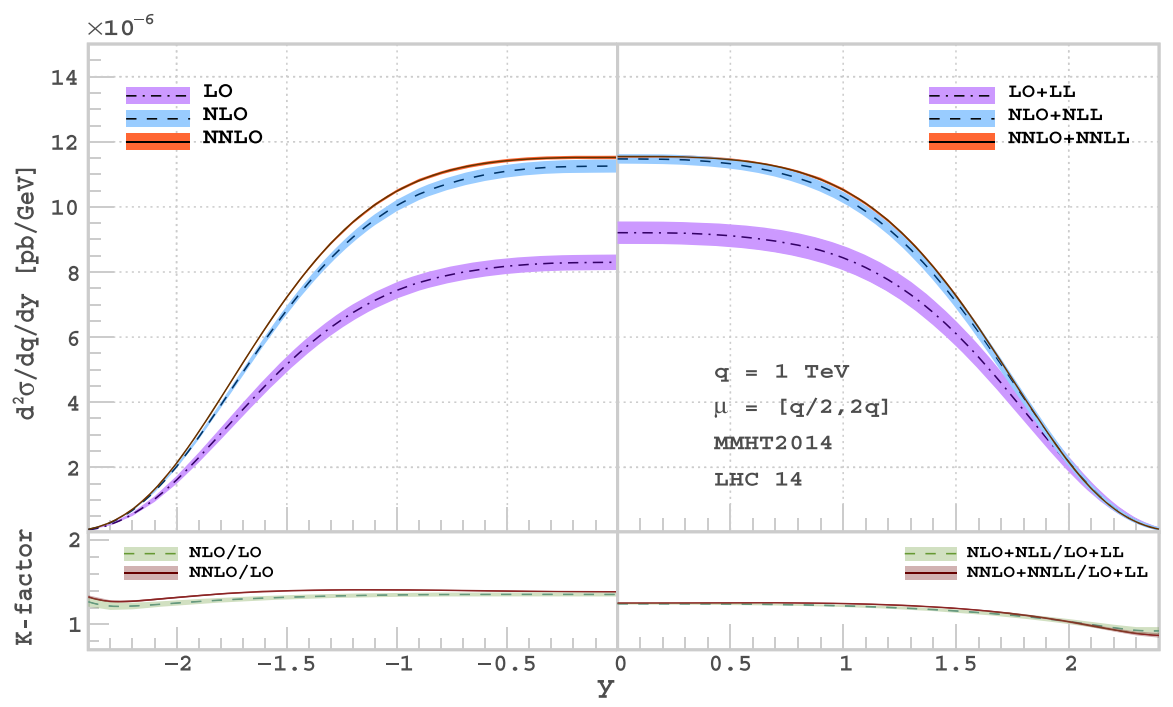

FIG. 6. Same as Fig. 4 but for $q=1 \mathrm{TeV}$. 


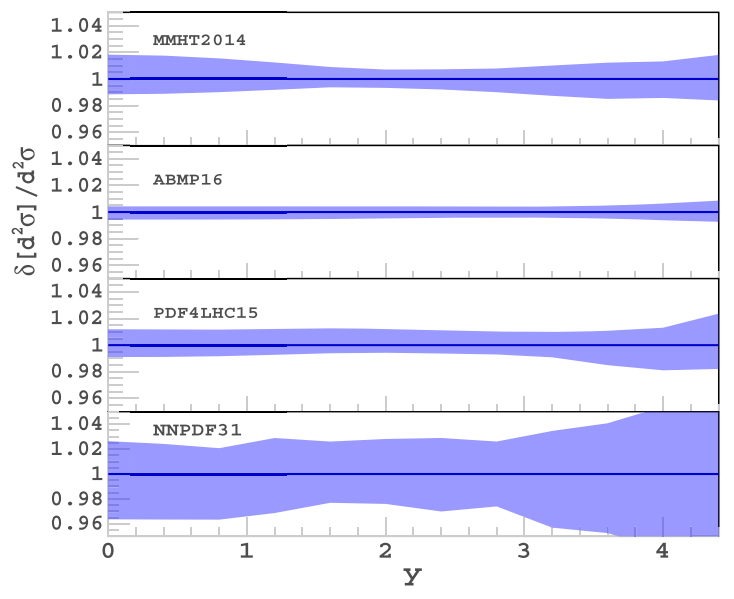

FIG. 7. PDF variation at NNLO + NNLL using various sets. The $y$-axis represents the ratio of extremum variation over the central PDF set.

two extreme scales are of opposite sign and their individual contributions are such that the NNLO + NNLL $(q \bar{q})$ curve shows a stable behavior as compared to NNLO $(q \bar{q})$. While the fixed order decreases by $2.36 \%$ from $M_{Z} / 2$ to $2 M_{Z}$, the corresponding decrease for NNLO + NNLL $(q \bar{q})$ is $1.53 \%$. This confirms the reduction of scale dependence upon adding resummed terms to the fixed order contributions. To estimate the percentage corrections purely coming from the threshold region from this channel at each order of the perturbation theory, we have considered the case where the central scale is chosen to be $\mu_{R}=\mu_{F}=M_{Z}$. As expected, at LO both fixed order and the truncated resummed predictions agree. But, at NLO and at NNLO we find truncated one is $7 \%-8 \%$ and $12 \%-13 \%$ larger compared to respective fixed order at the central rapidity region. The largeness of the truncated results gets compensated by the -ve corrections coming from other channels emerging at respective orders. However the scenario entirely reverses when we consider all the channels at NNLO. We find that the differential cross section at NNLO (all) increases by $0.29 \%$ in the entire range of $\mu_{R}$ values; the corresponding increase for NNLO + NNLL (all) is $1.29 \%$. This reduction of the scale dependence at NNLO is due to cancellations among different partonic channels. However the resummation effects come only from $q \bar{q}$ channel which adds to the fixed order in such a way that

TABLE III. Cross sections at NNLO + NNLL using different PDF sets along with percentage uncertainties for $y=0,0.8$, 1.6, 2.4.

\begin{tabular}{ccccc}
\hline \hline $\mathrm{y}$ & MMHT & ABMP & NNPDF & PDF4LHC \\
\hline 0.0 & $79.568_{-1.16 \%}^{+1.83 \%}$ & $79.756_{-0.56 \%}^{+0.43 \%}$ & $81.959_{-3.64 \%}^{+2.64 \%}$ & $78.734_{-0.89 \%}^{+1.20 \%}$ \\
0.8 & $78.340_{-0.99 \%}^{+1.55 \%}$ & $78.202_{-0.56 \%}^{+0.43 \%}$ & $80.256_{-3.66 \%}^{+2.07 \%}$ & $77.390_{-0.83 \%}^{+1.17 \%}$ \\
1.6 & $74.588_{-0.63 \%}^{+0.90 \%}$ & $73.738_{-0.52 \%}^{+0.42 \%}$ & $75.178_{-2.31 \%}^{+2.61 \%}$ & $73.505_{-0.61 \%}^{+1.26 \%}$ \\
2.4 & $67.985_{-0.79 \%}^{+0.72 \%}$ & $66.653_{-0.44 \%}^{+0.41 \%}$ & $67.354_{-3.01 \%}^{+2.89 \%}$ & $67.070_{-0.62 \%}^{+1.11 \%}$ \\
\hline \hline
\end{tabular}

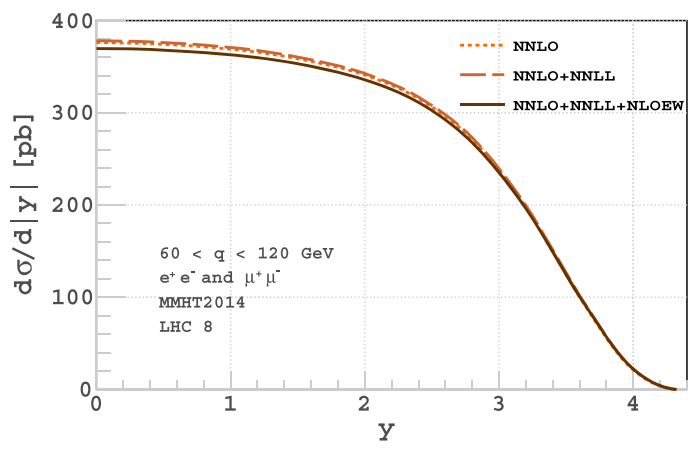

FIG. 8. Rapidity distribution at NNLO + NNLL for $8 \mathrm{TeV}$ LHC in the invariant mass range $60<q<120 \mathrm{GeV}$. The dotted line is the fixed order NNLO contribution, the dashed line represents NNLO + NNLL result and the solid line includes EW corrections.

the resummed uncertainty increases. This explains the increase of the scale uncertainty at each resummed order depicted in Fig. 4. Furthermore an incomplete cancellation of the factorization scale dependence against the PDFs which do not contain resummed threshold logarithms also increases the band. For the threshold resummation effects in PDFs, see [69]. For the fixed order, the K-factor at NLO varies between 1.3 and 1.2 and at NNLO between 1.37 and 1.3 over the entire rapidity region. On the other hand, the K-factors at both $\mathrm{NLO}+$ NLL and NNLO + NNLL significantly overlap with each other over most of the regions of rapidity and stay around 1.2. This demonstrates a better perturbative convergence for resummed case compared to the fixed order. In Table II, we have presented the cross section for benchmark rapidity values along with the percentage scale uncertainties. Note that the differential cross-section at NNLO + NNLL level for the central scale is well approximated by the same at $\mathrm{NLO}+\mathrm{NLL}$. In fact, NNLO + NNLL increases approximately by $0.8 \%$ with respect to NLO + NLL; the corresponding number for NNLO over NLO is approximately $3 \%$. From the trend that resummed results give, we anticipate $\mathrm{N}^{3} \mathrm{LO}+\mathrm{N}^{3} \mathrm{LL}$ cross-section will fall completely within the NNLO + NNLL band.

In Fig. 6, we have plotted both fixed order and resummed results at various orders for the larger invariant mass at $q=1 \mathrm{TeV}$. Interestingly, the uncertainty bands at NLO + NLL and NNLO + NNLL levels are better compared to those from fixed order. Also the predictions at various orders are closer compared to those from fixed order demonstrating better perturbative convergence of the higher order predictions from the resummed terms. In fact the resummed $\mathrm{K}$-factor for the central rapidity at NNLO + NNLL is 1.25 compared to 1.39 at NNLO.

As there are several PDF groups in the literature, each providing sets of PDFs, it is customary to estimate the uncertainty resulting from the choice of PDFs within each set of a given PDF group. Using PDFs from different PDF groups namely MMHT2014nnlo68cl [61], ABMP [70], 

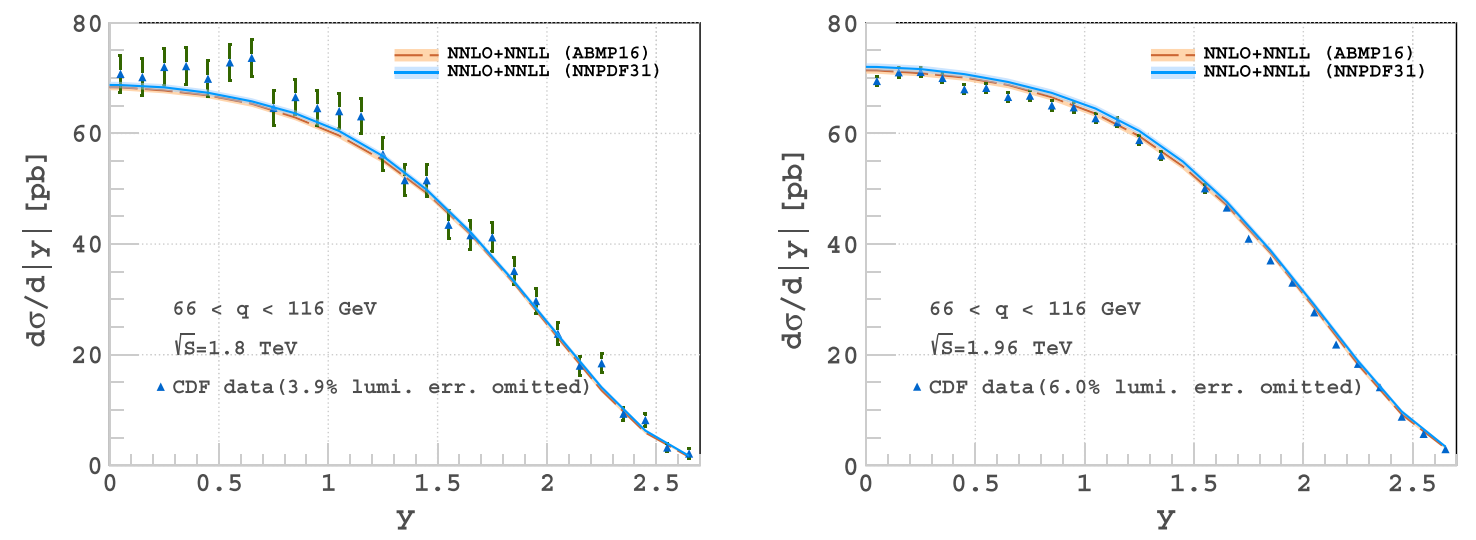

FIG. 9. Comparison between the resummed results and the CDF data $[80,81]$ at $\sqrt{s}=\{1.8 \mathrm{TeV}, 1.96 \mathrm{TeV}\}$ in the invariant mass range $66<q<116 \mathrm{GeV}$ for two different PDF sets.

NNPDF3.1 [71] and PDF4LHC [72] we have obtained the cross sections along with the corresponding PDF uncertainty. In Fig. 7, we have plotted the uncertainty bands for various PDF sets as function of rapidity in order to demonstrate the correlation of PDF uncertainty with the rapidity values. This will help to better constrain the PDF fits using measurements on rapidity in the Drell-Yan process. In Table III, we have also tabulated the cross sections along with \% uncertainties resulting from the choice of different PDFs.

We have studied the $q$-integrated rapidity distribution at the LHC with $8 \mathrm{TeV}$ center of mass energy at NNLO + NNLL. The invariant mass is integrated between $60 \mathrm{GeV}$ and $120 \mathrm{GeV}$ and choose $\mu_{R}=\mu_{F}=M_{Z}$. Unlike our earlier analysis, we have included both $e^{+} e^{-}$as well as $\mu^{+} \mu^{-}$final states. NLO EW corrections are also included as they are also comparable to QCD corrections at NNLO + NNLL. The EW contributions are obtained by using the publicly available code Horace-3.2 [73-76]. We use the $G_{\mu}$ scheme and take $G_{F}=1.16639 \times 10^{-5}$, $M_{W}=80.395 \mathrm{GeV}, M_{Z}=91.1876 \mathrm{GeV}$ and use $\mathrm{MMH}$ T2014nlo68cl pdf. The electron and muon masses are taken to be $m_{e}=0.51099 \mathrm{MeV}$ and $m_{\mu}=0.10566 \mathrm{GeV}$ respectively. The NNLL contribution increases the cross section by roughly around $0.5 \%$ with respect to NNLO. However the EW corrections at NLO give negative contribution to the cross-section. The corrections are different for $e^{+} e^{-}$ and $\mu^{+} \mu^{-}$pairs. For electrons, the EW contributions are twice that of muons. In total from the electron and muon channels, we see an overall $2.3 \%$ decrease in the cross section with respect to the NNLO in the central rapidity region. The rapidity distribution in Fig. 8 being inclusive in transverse momenta of the final state leptons, can not be directly compared with the results presented in [77] where a minimum transverse momenta cut is applied in the selection of final state leptons. To really compare this one needs distributions exclusive of transverse momenta which at the moment beyond the scope of the current paper and we leave it to future work.
Both at Tevatron and at the LHC, there are already precise measurements of rapidity distributions for different ranges of invariant mass $q$. For one of the earliest set of measurements see NuSea $[78,79]$. Since the data from the LHC depends heavily on the kinematic cuts of the final states we cannot directly compare against our predictions. On the other hand CDF $[80,81]$ has data for the rapidity distributions for wide range of $y$ with invariant mass range $66<q<116 \mathrm{GeV}$. In Fig. 9, we have compared our predictions against the data at $\sqrt{s}=1.8 \mathrm{TeV}$ and at $\sqrt{s}=$ $1.96 \mathrm{TeV}$ after integrating $q$ between the above mentioned range for two different choices of PDF sets. The scale uncertainty is obtained as before by using 7-point scale variations around the central value $\mu_{R}=\mu_{F}=M_{Z}$. We note that at NNLO + NNLL level, the resummed contributions over the fixed order is very mild, less than $0.5 \%$. We have also observed that the resummed effects become significant for large invariant mass regions.

\section{DISCUSSION AND CONCLUSION}

In this article we have done a detailed study on the role of resummed threshold logarithms for the rapidity distribution of pairs of leptons in the Drell-Yan process at the LHC. Being one of the cleanest channels at the hadron colliders, precise measurements of various observables such as inclusive cross section, transverse momentum and rapidity distributions are already available. Precise predictions from perturbative QCD are known to NNLO level for long and corrections from electroweak theory have become available in recent times. The latter effects being close to the second order effects from QCD so that dedicated efforts to understand the EW effects have been undertaken. Owing to the dominant QCD interactions, soft gluons play vital role in most of the observables. They show up in certain kinematic regions through large logarithms in the perturbative computations. Often they spoil the reliability of the fixed order predictions. In this present article, we have made a detail study on the effect of these soft gluons within the resummation framework. In the 
literature two different approaches exist. They differ in the kind of logarithms that are resummed to all orders. The approach which uses Mellin-Fourier transformation to achieve the resummation to resum large logarithms of the scaling variable $z$ has been well studied for the rapidity distribution. Threshold logarithms resulting from regions where the scaling variable $z_{i}$ approach unity are successfully resummed using the M-M approach. We used the latter approach to get the quantitative predictions at NNLO + NNLL level. Since these formalisms resum different type of logarithms to all orders, they are expected to give different numerical predictions. In this article, we have not only undertaken a detailed study on the numerical impact of the M-M approach for the first time for the DrellYan process but also made a detailed numerical comparison against the M-F approach. While at LL and NLL level, they differ very much, surprisingly at the NNLL level both the approaches converge to a few percent correction to the fixed order prediction. This could be accidental, however it is desirable to understand this coincidence at NNLL level. Our numerical study on the dependence of renormalization and factorization scales shows that the optimal central scales for the resummed result are $\mu_{R}=M_{Z} / 2$ and $\mu_{F}=$ $M_{Z}$ while it is $\mu_{R}=\mu_{F}=M_{Z}$ for NNLO. We have also found that, for wide range of rapidity, the scale uncertainties from NNLL contributions at every order are slightly larger than those from fixed order results. We believe that this could be due to an incomplete cancellation of scale dependent terms between resummed result and the PDFs. Note that the PDFs that we use are extracted from data using the fixed order perturbative predictions for the observables and also using evolutions equations controlled by splittings functions computed to desired order in strong coupling constant. Hence, we expect that there will be a better cancellation of scale if appropriate resummed PDF sets are available. We have also presented our predictions for various choices of PDFs from various PDF groups. Each group has several sets and hence we have not only made comparisons with respect to various groups but also estimated the uncertainty from different sets within each PDF group. We have also predicted the $q$ integrated rapidity distribution. Since our resummation formalism cannot take into the experimental cuts such as the transverse momentum and/or polar angles of the final state leptons, we can not make any direct comparisons with the existing data on the $\mathrm{q}$ integrated rapidity distribution measured at the LHC which are extracted after employing cuts on transverse momentum of final state leptons. On the other hand we have compared our predictions against $\mathrm{CDF}$ data at Tevatron for the invariant mass range $66<q<116 \mathrm{GeV}$ and found good agreement within both theoretical and experimental uncertainties. We believe that perturbative results that take into account both fixed order as well as the resummed contributions will provide a precise determination of PDFs from the ample data that are already available at the LHC.

\section{ACKNOWLEDGMENTS}

We thank S. Alekhin, J. Blümlein, S. Moch, and F. J. Tackmann for suggestions and careful reading of the manuscript. We are thankful to S. Catani, M. Grazzini, M. Neubert, G. Ferrera, A. Vicini, M. Bonvini, and L. Rotolli, W. Vogelsang for useful discussions. G. D. would like to thank J. Michel and L. Dixon for fruitful discussions. P. K. D. would like to thank G. Ridolfi, R. Harlander, and F. Maltoni for useful suggestions to improve the presentation of the paper. P. B. would like to thank T. Becher and L. Magnea for useful discussions. Ravindran would like to thank M. Neubert for his kind hospitality at University of Mainz where part of the work was carried out. We also thank P. Mangalapandi for his help related to cluster computing system at IMSc. G. D. acknowledges research support from DESY.
[1] S. Catani and L. Trentadue, Nucl. Phys. B327, 323 (1989).

[2] V. Ravindran, J. Smith, and W. L. van Neerven, Nucl. Phys. B767, 100 (2007).

[3] P. Banerjee, G. Das, P. K. Dhani, and V. Ravindran, Phys. Rev. D 97, 054024 (2018).

[4] S. D. Drell and T.-M. Yan, Phys. Rev. Lett. 25, 316 (1970); 25, 902(E) (1970).

[5] V. A. Khoze, A. D. Martin, R. Orava, and M. G. Ryskin, Eur. Phys. J. C 19, 313 (2001).

[6] G. Altarelli, R. K. Ellis, and G. Martinelli, Nucl. Phys. B143, 521 (1978); B146, 544(E) (1978).

[7] G. Altarelli, R. K. Ellis, and G. Martinelli, Nucl. Phys. B157, 461 (1979).
[8] R. Hamberg, W. L. van Neerven, and T. Matsuura, Nucl. Phys. B359, 343 (1991); B644, 403(E) (2002).

[9] R. V. Harlander and W. B. Kilgore, Phys. Rev. Lett. 88, 201801 (2002).

[10] T. Ahmed, M. Mahakhud, N. Rana, and V. Ravindran, Phys. Rev. Lett. 113, 112002 (2014).

[11] Y. Li, A. von Manteuffel, R. M. Schabinger, and H. X. Zhu, Phys. Rev. D 90, 053006 (2014).

[12] S. Dittmaier and M. Kramer, Phys. Rev. D 65, 073007 (2002).

[13] U. Baur, O. Brein, W. Hollik, C. Schappacher, and D. Wackeroth, Phys. Rev. D 65, 033007 (2002).

[14] C. Anastasiou, L. J. Dixon, K. Melnikov, and F. Petriello, Phys. Rev. Lett. 91, 182002 (2003). 
[15] C. Anastasiou, L. J. Dixon, K. Melnikov, and F. Petriello, Phys. Rev. D 69, 094008 (2004).

[16] S. Catani, L. Cieri, G. Ferrera, D. de Florian, and M. Grazzini, Phys. Rev. Lett. 103, 082001 (2009).

[17] K. Melnikov and F. Petriello, Phys. Rev. D 74, 114017 (2006).

[18] R. Gavin, Y. Li, F. Petriello, and S. Quackenbush, Comput. Phys. Commun. 184, 209 (2013).

[19] V. Ravindran and J. Smith, Phys. Rev. D 76, 114004 (2007).

[20] T. Ahmed, M. K. Mandal, N. Rana, and V. Ravindran, Phys. Rev. Lett. 113, 212003 (2014).

[21] Y. Li and F. Petriello, Phys. Rev. D 86, 094034 (2012).

[22] S. Frixione and B. R. Webber, J. High Energy Phys. 06 (2002) 029.

[23] S. Frixione, P. Nason, and C. Oleari, J. High Energy Phys. 11 (2007) 070.

[24] J. Alwall, R. Frederix, S. Frixione, V. Hirschi, F. Maltoni, O. Mattelaer, H. S. Shao, T. Stelzer, P. Torrielli, and M. Zaro, J. High Energy Phys. 07 (2014) 079.

[25] R. K. Ellis, G. Martinelli, and R. Petronzio, Nucl. Phys. B211, 106 (1983).

[26] P. B. Arnold and M. H. Reno, Nucl. Phys. B319, 37 (1989); B330, 284(E) (1990).

[27] R. J. Gonsalves, J. Pawlowski, and C.-F. Wai, Phys. Rev. D 40, 2245 (1989).

[28] E. Mirkes, Nucl. Phys. B387, 3 (1992).

[29] E. Mirkes and J. Ohnemus, Phys. Rev. D 51, 4891 (1995).

[30] A. Accardi et al., Eur. Phys. J. C 76, 471 (2016).

[31] I. Scimemi and A. Vladimirov, Eur. Phys. J. C 78, 89 (2018).

[32] I. Scimemi and A. Vladimirov, J. High Energy Phys. 08 (2018) 003.

[33] G. F. Sterman, Nucl. Phys. B281, 310 (1987).

[34] S. Catani and L. Trentadue, Nucl. Phys. B353, 183 (1991).

[35] S. Moch and A. Vogt, Phys. Lett. B 631, 48 (2005).

[36] E. Laenen and L. Magnea, Phys. Lett. B 632, 270 (2006).

[37] A. Idilbi, X.-d. Ji, J.-P. Ma, and F. Yuan, Phys. Rev. D 73, 077501 (2006).

[38] V. Ravindran, Nucl. Phys. B746, 58 (2006).

[39] J. C. Collins, D. E. Soper, and G. F. Sterman, Nucl. Phys. B250, 199 (1985).

[40] S. Catani, D. de Florian, and M. Grazzini, Nucl. Phys. B596, 299 (2001).

[41] A. Idilbi and X.-d. Ji, Phys. Rev. D 72, 054016 (2005).

[42] T. Becher and M. Neubert, Eur. Phys. J. C 71, 1665 (2011).

[43] D. Westmark and J. F. Owens, Phys. Rev. D 95, 056024 (2017).

[44] E. Laenen and G. F. Sterman, in The Fermilab Meeting DPF 92. Proceedings, 7th Meeting of the American Physical Society, Division of Particles and Fields, Batavia, USA, 1992. Vol. 1, 2 (1992), p. 987.

[45] G. F. Sterman and W. Vogelsang, J. High Energy Phys. 02 (2001) 016.

[46] A. Mukherjee and W. Vogelsang, Phys. Rev. D 73, 074005 (2006).

[47] P. Bolzoni, Phys. Lett. B 643, 325 (2006).

[48] M. Bonvini, S. Forte, G. Ridolfi, and L. Rottoli, J. High Energy Phys. 01 (2015) 046.
[49] T. Becher and M. Neubert, Phys. Rev. Lett. 97, 082001 (2006).

[50] T. Becher, M. Neubert, and G. Xu, J. High Energy Phys. 07 (2008) 030.

[51] M. Bonvini, S. Forte, and G. Ridolfi, Nucl. Phys. B847, 93 (2011).

[52] T. Ahmed, M. K. Mandal, N. Rana, and V. Ravindran, J. High Energy Phys. 02 (2015) 131.

[53] G. P. Korchemsky and A. V. Radyushkin, Nucl. Phys. B283, 342 (1987).

[54] G. P. Korchemsky, Phys. Lett. B 220, 629 (1989).

[55] I. A. Korchemskaya and G. P. Korchemsky, Phys. Lett. B 287, 169 (1992).

[56] J. Kodaira and L. Trentadue, Phys. Lett. 112B, 66 (1982).

[57] S. Catani, E. D’Emilio, and L. Trentadue, Phys. Lett. B 211, 335 (1988).

[58] S. Moch, J. A. M. Vermaseren, and A. Vogt, Nucl. Phys. B688, 101 (2004).

[59] S. Catani, D. de Florian, M. Grazzini, and P. Nason, J. High Energy Phys. 07 (2003) 028.

[60] O. V. Tarasov, A. A. Vladimirov, and A. Y. Zharkov, Phys. Lett. 93B, 429 (1980).

[61] L. A. Harland-Lang, A. D. Martin, P. Motylinski, and R. S. Thorne, Eur. Phys. J. C 75, 204 (2015).

[62] A. Buckley, J. Ferrando, S. Lloyd, K. Nordstrm, B. Page, M. Rfenacht, M. Schnherr, and G. Watt, Eur. Phys. J. C 75, 132 (2015).

[63] M. Bonvini, https://www.ge.infn.it/ bonvini/redy.

[64] S. Catani, M. L. Mangano, P. Nason, and L. Trentadue, Nucl. Phys. B478, 273 (1996).

[65] D. P. Anderle, F. Ringer, and W. Vogelsang, Phys. Rev. D 87, 034014 (2013).

[66] M. A. Ebert, J. K. L. Michel, and F. J. Tackmann, J. High Energy Phys. 05 (2017) 088.

[67] L. Dixon, http://www.slac.stanford.edu/ lance/Vrap.

[68] R. D. Ball, L. Del Debbio, S. Forte, A. Guffanti, J. I. Latorre, J. Rojo, and M. Ubiali, Nucl. Phys. B838, 136 (2010).

[69] M. Bonvini, S. Marzani, J. Rojo, L. Rottoli, M. Ubiali, R. D. Ball, V. Bertone, S. Carrazza, and N. P. Hartland, J. High Energy Phys. 09 (2015) 191.

[70] S. Alekhin, J. Blmlein, S. Moch, and R. Placakyte, Phys. Rev. D 96, 014011 (2017).

[71] R. D. Ball et al. (NNPDF), Eur. Phys. J. C 77, 663 (2017).

[72] J. Butterworth et al., J. Phys. G 43, 023001 (2016).

[73] https://www2.pv.infn.it/ hepcomplex/horace.html.

[74] S. Alioli et al., Eur. Phys. J. C 77, 280 (2017).

[75] C. M. Carloni Calame, G. Montagna, O. Nicrosini, and A. Vicini, J. High Energy Phys. 10 (2007) 109.

[76] C. M. Carloni Calame, G. Montagna, O. Nicrosini, and M. Treccani, J. High Energy Phys. 05 (2005) 019.

[77] V. Khachatryan et al. (CMS), Eur. Phys. J. C 75, 147 (2015).

[78] R. S. Towell et al. (NuSea), Phys. Rev. D 64, 052002 (2001).

[79] J. C. Webb, Ph.D. thesis, New Mexico State University, 2003, arXiv:hep-ex/0301031.

[80] T. Affolder et al. (CDF), Phys. Rev. D 63, 011101 (2000).

[81] T. A. Aaltonen et al. (CDF), Phys. Lett. B 692, 232 (2010). 